\title{
Tip influence on plasmon excitations in single gold particles in an STM
}

\author{
N. Nilius, ${ }^{*}$ N. Ernst, and H.-J. Freund \\ Fritz-Haber-Institut der Max-Planck-Gesellschaft, Faradayweg 4-6, D 14195 Berlin, Germany
}

(Received 4 September 2001; published 8 March 2002)

\begin{abstract}
Photon-emission spectra have been measured for individual titania-supported gold particles excited by the electron current from an STM tip. Depending on the interaction strength between tip and cluster, the emission behavior changes from excitations with Mie-plasmon character at $2.3 \mathrm{eV}$ to modes of tip-induced plasmons at $1.8 \mathrm{eV}$. The strong tip influence on plasmon excitations in Au particles results from the almost constant dielectric function of gold between 1.8 and $2.3 \mathrm{eV}$, making the plasmon resonance sensitive to small changes in the electromagnetic environment. The lifetime of collective modes, derived from homogeneous linewidths, is governed by dielectric losses in gold, in particular, by gold interband transitions.
\end{abstract}

DOI: 10.1103/PhysRevB.65.115421

PACS number(s): 73.22.Lp, 78.67.Bf, 68.37.Ef, 68.47.Jn

\section{INTRODUCTION}

The electronic and optical properties of small noble-metal clusters are dominated by strong collective electronic excitations, well known as Mie plasmons. ${ }^{1}$ The plasmon can be viewed as coherent oscillations of the conduction electrons relative to the immobile ion cores in the particle. Its resonance frequency sensitively depends on material, size, and shape of the cluster and interactions with the surrounding medium. A controlled modification of these parameters enables a shift of the plasmon resonance position from the visible to the near-ultraviolet spectral range. This variability explains the wide use of noble-metal clusters in different technological applications, for example, in optical and polarization filters, nonlinear optical devices or stained glass. ${ }^{2-5}$

From the experimental point of view, the investigation of Mie plasmons in small metal aggregates still causes a number of difficulties, mostly connected with the limited lateral resolution of conventional optical and electron spectroscopies. Since a certain size distribution in cluster ensembles on surfaces or matrices cannot be avoided, conventional experimental methods usually give averaged values on energy position and width of the plasmon resonance. A direct correlation between size and shape of a cluster and its optical behavior demands for a local spectroscopy, which allows the examination of individual metal particles. The combination of scanning tunneling microscopy and photon-emission spectroscopy (PSTM) represents a promising experimental approach to fulfill these requirements. ${ }^{6,7}$ The method benefits from the possibility of exciting the plasmon by electron injection into a cluster and from the enhanced probability for radiating decays of the collective oscillation. The high localization of the electron beam from an STM tip enables the controlled excitation of individual particles. The spectroscopic analysis of photons emitted after electron injection gives the requested properties of the Mie plasmon. Because of the fixed tip-sample geometry in an STM, electron injection into a cluster exclusively excites the plasmon mode oscillating perpendicular to the sample surface ( 1,0 mode). The parallel oscillation (1,1 mode), normally lower in energy, cannot be observed within these experiments. The STMmediated investigation of plasmon excitations in small metal particles has been successfully employed on different clustersubstrate combinations, e.g., on silver particles on $\mathrm{Si}(111)$ (Refs. 8 and 9), $\mathrm{TiO}_{2}$ (110) (Ref. 10), and $\mathrm{Al}_{2} \mathrm{O}_{3} / \mathrm{NiAl}(110$ ) (Ref. 11) or gold clusters on $\mathrm{Si}(111)$ (Refs. 9 and 12) and $\mathrm{Al}_{2} \mathrm{O}_{3} / \mathrm{NiAl}(110)$ (Ref. 13).

The advantage of a high lateral resolution in these experiments has, however, to be paid by an additional influence of the STM tip on the cluster-excitation process. ${ }^{7,14}$ The proximity of tip and cluster changes the electromagnetic potential "felt" by the plasmon and shifts the energy position of the collective mode. Two effects of tip-sample interactions have to be considered.

(i) The static electric field of the tunnel bias in the range of some $\mathrm{V} / \mathrm{nm}$ superimposes an additional restoring force on the oscillating plasmon dipole. Additionally, the screening of this electric field at the cluster surface induces slight deviations from the equilibrium electron density in the particle. The role of electrostatic effects on the plasmon dipole can be investigated by modifying bias and polarity in the STM cavity.

(ii) Dynamic electromagnetic interactions between tip and sample in an STM may also affect the Mie plasmon in a metal particle. The oscillating dipole in the cluster induces an image dipole in the metallic tip, depending on polarizability and dielectric losses of the tip material. The alignment and phase shift between the dipoles determine the strength of dipole-dipole interactions and, therefore, resonance energy and lifetime of the Mie plasmon in the cluster.

The importance of tip effects on plasmon excitations becomes evident for light-emission processes from planar metal surfaces, probed with an STM tip. Whereas surface plasmons in well isolated, flat electrodes do not couple to light fields because of parallel-momentum conservation, the electromagnetic interaction between tip and sample in an STM causes an excitation of radiating interface plasmons. ${ }^{15}$ These tip-induced plasmons (TIP's) are characterized by coherent oscillations of the coupled electron gases in tip and sample, driven by inelastically tunneling electrons. ${ }^{16}$

Two borderline cases have, therefore, to be considered, when dealing with light-emission processes in an STM: (i) Mie plasmons in supported metal particles, nearly unaffected 
by the presence of the STM tip and (ii) tip-induced plasmons excited in the cavity due to enhanced tip-sample interactions. The following paper presents light-emission spectra obtained from individual titania-supported gold particles in an STM cavity. The emission behavior as a function of cluster size is used as a measure of tip-sample coupling in the STM. Gold particles have been chosen because of a particularity in the frequency dependence of their dielectric function, making the energy position of plasmon modes sensitive to small changes in the electromagnetic environment of the cluster. On the other side, the system $\mathrm{Au} / \mathrm{TiO}_{2}$ has gained increasing interest because of its high catalytic activity for $\mathrm{CO}$ oxidation at low temperatures (Refs. 17 and 18). There have been studies to connect the catalytic properties of small $\mathrm{Au}$ particles with their electronic structure, especially the metal to nonmetal transition, both from the experimental ${ }^{19}$ and theoretical point of view. ${ }^{20}$

\section{EXPERIMENT}

The experiments have been carried out in an UHV chamber $\left(p<2 \times 10^{-10} \mathrm{mbar}\right)$, equipped with a Beetle-type STM and standard surface-science methods for sample preparation and analysis. The STM head is surrounded by a parabolic mirror, which collects photons emitted from the tunnel junction. After passing a quartz window, the light is focused on the entrance slit of a grating spectrograph and detected with a liquid nitrogen cooled charge coupled device outside the vacuum chamber. The optical arrangement allows the parallel detection of emission spectra in an energy range between 1 and $6 \mathrm{eV}$. Details of the experimental setup have been described earlier. ${ }^{21}$ Light emission from selected gold particles is excited by electron injection from the STM tip, electrochemically etched from a polycrystalline tungsten wire. The STM is operated in the tunneling or near field-emission regime at $U_{\text {gap }}<20 \mathrm{~V}$, which reduces tip-sample distances to less than $2 \mathrm{~nm}$. At these conditions, the excitation of an individual particle is secured. For typical electron currents of $2 \mathrm{nA}$, a light accumulation time of $500 \mathrm{~s}$ is required to obtain photon-emission spectra with acceptable signal to noise ratio.

A $\mathrm{TiO}_{2}$ single crystal, oriented in [110] direction, was used as substrate for the gold particles. Prior to STM experiments, a sufficient conductivity was adjusted in the $\mathrm{TiO}_{2}$ sample by reducing the oxide at $1000 \mathrm{~K}$ in $\mathrm{UHV}^{22}$ The reduction procedure could be followed by monitoring changes in the crystal color from transparent to blue. A clean (110) surface was produced by alternating cycles of Ar sputtering and annealing to $800 \mathrm{~K}$. Completing these preparation steps, STM images of the surface showed large terraces covered with parallel white lines [Fig. 1(a)]. This characteristic line pattern in connection with $(2 \times 1)$-superstructure reflexes in low-energy electron diffraction indicates the formation of an added row reconstruction on the reduced $\mathrm{TiO}_{2}$ (110) surface. ${ }^{23,24}$ Gold clusters were prepared by atom deposition from the gas phase, followed by diffusion and nucleation to three-dimensional particles at room temperature. Oxide step edges served as preferential nucleation centers, leading to a mean cluster density of $4 \times 10^{-11} \mathrm{~cm}^{-2}$. Figures $1(\mathrm{~b})-1(\mathrm{~d})$
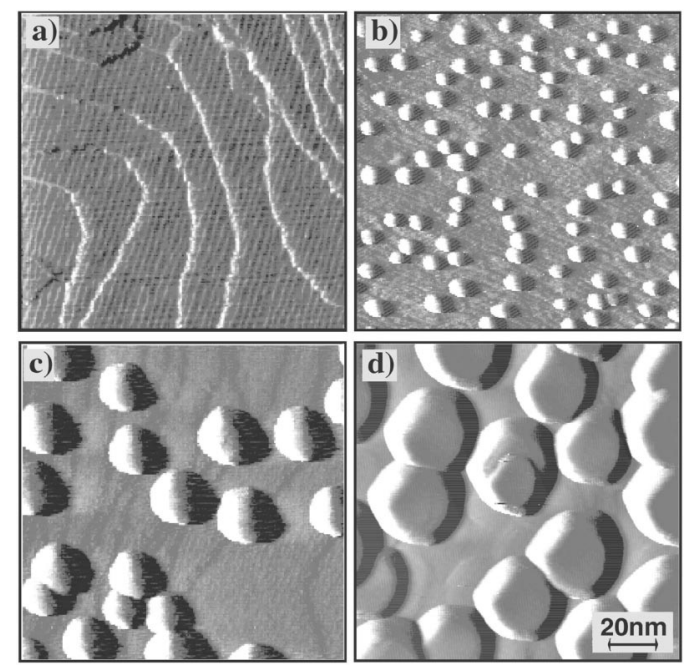

FIG. 1. STM images of the clean $\mathrm{TiO}_{2}$ (110) surface (a) and $\mathrm{Au}$ clusters on $\mathrm{TiO}_{2}$ (110) for different metal exposures: 0.5 ML (b), 2 ML (c), and $5 \mathrm{ML}$, (d). Images have been obtained at $-2 \mathrm{~V}$ tip bias and $0.5 \mathrm{nA}$ tunnel current. Image sizes are $130 \times 130 \mathrm{~nm}$.

show STM topographies of the surface after exposure of 0.5 , 2.0, and 5.0 monolayers (ML) of gold, estimated from ex situ quartz balance measurements. Cluster sizes from STM images have been corrected for the effect of tip convolution. For this purpose, the apparent broadening of substrate step edges was taken from STM topographies to deduce a mean radius of the tip apex.

\section{RESULTS AND DISCUSSION}

\section{A. Photon emission from Au particles on $\mathrm{TiO}_{2}$ : General behavior}

Emission spectra were measured for single gold particles selected from topographic STM images. Morphological changes of the particles or drift of the tip position during the measurements could be excluded through repeated imaging of the surface region after each spectroscopic run. No photon emission was detected from clean $\mathrm{TiO}_{2}$ at tip voltages below $\pm 20 \mathrm{~V}$ (Fig. 2 lower spectrum). This experimental result has to be explained by the absence of dipole-allowed transitions between the oxide band edges, responsible for a small cross section of radiating electron-hole-pair recombinations via the gap. ${ }^{25}$ However, the injection of electrons into an Au particle led to the occurrence of a strong emission line in the spectra. Peak positions were randomly distributed in a spectral range between 1.6 and $2.3 \mathrm{eV}$ and did not depend on the particle diameter alone. Even subsequent experiments on the same $\mathrm{Au}$ cluster resulted in different emission maxima. These instabilities in the photon-emission behavior could not be controlled by the excitation conditions of the cluster in the STM, such as tip bias or tunnel current. Moreover, they appeared as a consequence of accidental changes in the microscopic tip configuration, for instance, due to material transport between tip and sample. Fig. 2 shows emission spectra for two differently sized Au clusters on $\mathrm{TiO}_{2}(110)$, with a cluster diameter of $12 \mathrm{~nm}$ [spectra (i) and (iii)] and $8 \mathrm{~nm}$, respectively [spectra (ii) and (iv)]. After completing measurement (i) and (ii), a 


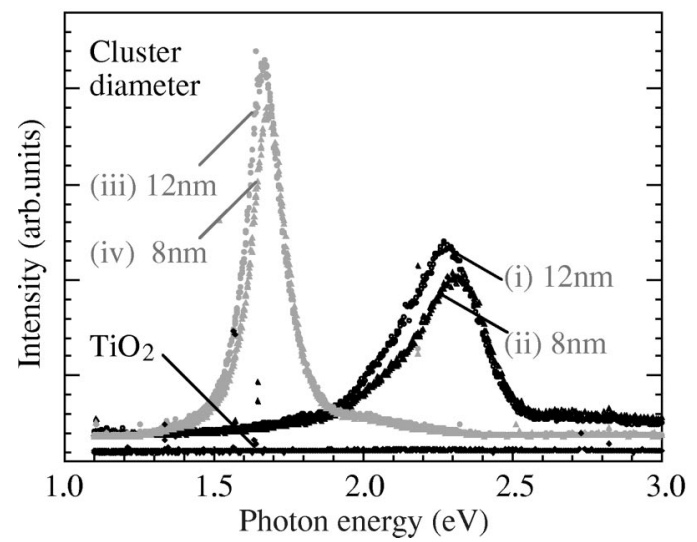

FIG. 2. Photon-emission spectra of two Au particles on $\mathrm{TiO}_{2}$ $\left(U_{\text {tip }}=-5 \mathrm{~V}, I=1.5 \mathrm{nA}\right)$. Cluster diameters were (i) 12 and (ii) 8 $\mathrm{nm}$. Spectra (iii) and (iv) were obtained on the same particles after material transfer to the STM tip.

tip change occurred, manifested in an enhanced lateral resolution power of the tip. As a consequence, repeated spectroscopic runs on the same particles gave different peak positions, redshifted from 2.28 to $1.67 \mathrm{eV}$ for the larger cluster [spectra (i) and (iii)] and from 2.31 to $1.68 \mathrm{eV}$ for the smaller one [spectra (ii) and (iv)].

Comparable shifts of peak energies have been observed in a number of different experiments, independently of cluster diameter or tunneling conditions. However, emission peaks from $\mathrm{Au}$ particles on $\mathrm{TiO}_{2}$ remained localized in the spectral range between 1.6 and $2.3 \mathrm{eV}$.

\section{B. Energy positions of the emission peaks}

Two mechanisms are conceivable as explanation for light emission from supported Au particles, localized in the tunnel junction of an STM. Neglecting the influence of the STM tip, electron injection into a cluster excites perpendicular 1,0 modes of the Mie plasmon, to be viewed as collective oscillations of the gold electron gas along the tip-sample axis. ${ }^{1}$ Its energy position can be estimated by inserting the dielectric functions of particle ${ }^{26}\left[\varepsilon_{\mathrm{Au}}(\omega)\right]$ and surrounding $\left(\varepsilon_{m}\right)$ into the resonance condition: ${ }^{27}$

$$
\varepsilon_{\mathrm{Au}}(\omega)=\varepsilon_{m} \frac{1-L_{\perp}}{L_{\perp}} .
$$

In Eq. (1), $L_{\perp}$ is the depolarization factor, accounting for the shape influence on the cluster polarizability perpendicular to the substrate. For a cluster height to diameter ratio of $0.5-$ 0.6 , as derived from STM images, a depolarization factor of 0.4 has to be used in the calculation. The cluster environment consists of $65 \%$ vacuum $\left(\varepsilon_{\mathrm{vac}}=1.0\right)$ and $35 \% \mathrm{TiO}_{2}(110)$ interface $^{28}(\varepsilon=6.48)$, yielding an averaged dielectric constant of $\varepsilon_{m}=2.95$. Inserting these values, a resonance position of $2.38 \mathrm{eV}$ is obtained for the 1,0 Mie mode in small gold clusters on $\mathrm{TiO}_{2}$. The calculated plasmon energy is centered in the upper range of the spectral region, where photonemission peaks from Au particles have been observed in present STM experiments. An extension of the resonance condition in Eq. (1), according to Ref. 29, allows the mod-

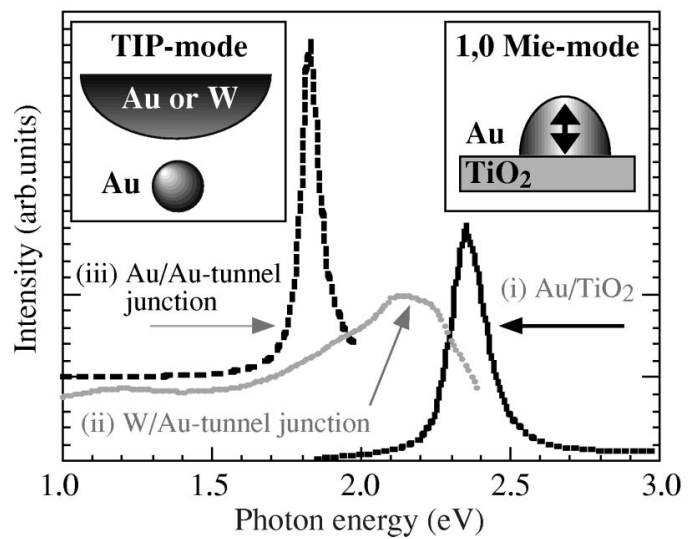

FIG. 3. Photon emission spectra of: (i) Mie plasmons in $\mathrm{Au}$ particles on $\mathrm{TiO}_{2}$, (ii) TIP modes in a W/Au and (iii) $\mathrm{Au} / \mathrm{Au}$ tunnel junction, calculated for $-5 \mathrm{~V}$ tip bias and $2 \mathrm{nA}$ electron current. The cluster diameter was set to $15 \mathrm{~nm}$. Corresponding emission mechanisms are illustrated in the insets.

eling of plasmon-induced absorption. A corresponding spectrum for $\mathrm{Au} / \mathrm{TiO}_{2}$ is shown in Fig. 3, curve (i).

A consideration of the STM tip in the theoretical treatment of the Mie-plasmon affects the resonance position of the collective mode in an Au particle. In a simplified picture, the oscillating plasmon dipole $\left(p_{\text {Mie }}\right)$ induces an image dipole in the metallic tip $\left(p_{\text {Tip }}\right)$, depending on the polarizability of the tip material. ${ }^{714}$ For a parallel orientation of the two dipoles, the attractive dipole-dipole interaction leads to a decreasing resonance frequency of the plasmon. The magnitude of this effect can be estimated, using the following equation:

$$
\begin{aligned}
\hbar \omega_{\text {Tip-Samp }}= & \hbar \omega_{\text {Mie }}+\frac{p_{\text {Mie }} p_{\text {Tip }}}{r_{\text {Tip-Samp }}^{3}} \\
& -\frac{3\left(p_{\text {Mie }} r_{\text {Tip-Samp }}\right)\left(p_{\text {Tip }} r_{\text {Tip-Samp }}\right)}{r_{\text {Tip-Samp }}^{5}} \\
= & \hbar \omega_{\text {Mie }}-\frac{2\left|p_{\text {Mie }}\right|\left|p_{\text {Tip }}\right|}{r_{\text {Tip-Samp }}^{3}} .
\end{aligned}
$$

Consequently, a decreasing plasmon energy, implying a redshift of the photon-emission peak, is favored by a strong induced tip dipole and a short tip-sample separation $\left(r_{\text {Tip-Samp }}\right)$. The limit of the tip-induced energy shift is given by the resonance position of the tip induced plasmon (TIP), whereby the oscillator strength of the coupled dipoles is almost equally stored in the electron gases of tip and sample. ${ }^{16}$ In this case, the polarizability of the system is not dominated by the cluster polarizability alone and the resonance position of the TIP mode is determined by the dielectric properties of tip and sample as well.

The energy of the TIP can be calculated by solving the Poisson equation in a nonretarded limit for the given tipsample geometry in an STM. A detailed description of the theoretical model can be found in the literature. ${ }^{16,30,31}$ Assuming a tip radius of $50 \mathrm{~nm}$, much larger than the mean radius of gold particles $(10 \mathrm{~nm})$, the actual geometry is approximated by a semi-infinite metal plane in proximity to a 
gold sphere. At these conditions, the electromagnetic response function in the tunnel cavity is calculated by using experimental dielectric functions to describe material properties of tip and cluster. ${ }^{26}$ The presence of the $\mathrm{TiO}_{2}$ support is neglected. Tip-cluster distances are determined by solving the Schrödinger equation for a one-dimensional tunnel barrier, treating tip and sample in a free electron-gas model. Values for tip bias and tunnel current are adapted to experimental conditions. Due to strong interactions between tip and sample in an STM, a dynamic electromagnetic field is induced in the tunnel cavity, driving TIP's in the electron gases of both electrodes. Radiating decays of the TIP's give rise to the emission of photons with a frequency distribution corresponding to the plasmon energy.

Figure 3, curve (ii) shows a calculated emission spectrum of the TIP mode, excited in the tunnel junction between $\mathrm{W}$ tip and $\mathrm{Au}$ cluster. The data have been computed with the following parameters: $U_{\text {tip }}=-5 \mathrm{~V}, I=2 \mathrm{nA}$, cluster diameter $15 \mathrm{~nm}$. The emission is dominated by a broad feature at $2.1 \mathrm{eV}$, indicating the resonance position of the W/Au plasmon mode. Additionally, a possible transfer of Au particles from the sample to the tip can be introduced in the TIP calculations. For this purpose, the tip material has to be replaced by the dielectric function of gold. Such a material transport has repeatedly been observed in the experiments and was associated with variations in the emission characteristics from $\mathrm{Au} / \mathrm{TiO}_{2}$. The calculated emission behavior of a tip-induced plasmon in the $\mathrm{Au} / \mathrm{Au}$ tunnel cavity is shown in Fig. 3, curve (iii). As a consequence of the high polarizability and small dielectric losses in gold compared to tungsten, the plasmon-mediated-emission peak is redshifted to $1.8 \mathrm{eV}$ and increased in intensity. Calculated peak positions from TIP modes in the W/Au or Au/Au tunnel junction lie in the lower spectral range, where light emission from Au particles has been observed in the present PSTM experiment.

Putting together what we have found so far, enables a consistent interpretation of STM-mediated, photon-emission processes from $\mathrm{Au}$ clusters on $\mathrm{TiO}_{2}$. Emission peaks centered at around $2.3 \mathrm{eV}$, have to be viewed as radiating decays of Mie-like plasmons in the supported Au particles. Their resonance frequencies are slightly modified by the electromagnetic coupling to the STM tip and correspond approximately to calculated plasmon energies for an undisturbed cluster-substrate system. Changes of the tip material enhance the importance of tip-cluster interactions and modify the nature of collective electronic excitations in the STM cavity towards plasmons with stronger TIP character. In particular, the observed emission peaks between 1.6 and $1.8 \mathrm{eV}$ can be understood as radiating decays of $\mathrm{Au} / \mathrm{Au}$ tip-induced plasmons, excited after a transfer of gold particles from the sample to the tip apex. Therefore, the broad distribution of emission peaks around $2 \mathrm{eV}$ is the consequence of a limited control over the microscopic configuration of the STM tip and no intrinsic property of the gold particles.

A comparable influence of the tip on the energy position of collective electronic excitations has not been observed for other cluster-substrate combinations, investigated by photon STM to our knowledge. Photon-emission peaks from individual $\mathrm{Ag}$ clusters, supported on $\mathrm{TiO}_{2}$ (Ref. 10) or $\mathrm{Al}_{2} \mathrm{O}_{3},{ }^{11}$

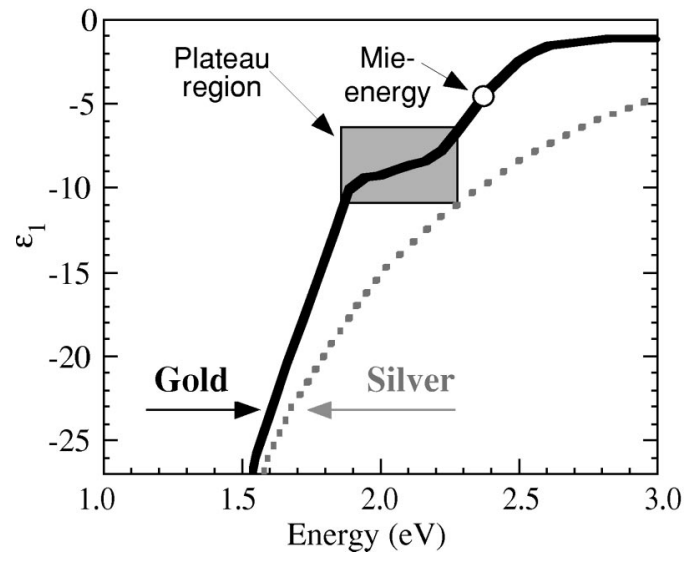

FIG. 4. Real part of the dielectric function for gold and silver from Ref. 26.

are concentrated in a narrow spectral window of approximately $0.1 \mathrm{eV}$. Furthermore, the peak positions are strongly correlated with cluster size. Also for small Au particles on $\mathrm{Al}_{2} \mathrm{O}_{3} / \mathrm{NiAl}(110)$, PSTM measurements showed photonemission peaks centered around $2.5 \mathrm{eV} .{ }^{13}$ In both cases, the energy position of observed emission peaks corresponds to the resonance frequency of Mie plasmons in the idealized cluster-substrate system and is not noticeably perturbed by interactions with the STM tip. The enhanced tip-cluster coupling for $\mathrm{Au} / \mathrm{TiO}_{2}$ appears to be an exceptional property of this material combination. An explanation for this emission behavior can be found in the specific frequency dependence of the gold dielectric function. As shown in Fig. 4, its real part, $\varepsilon_{1}(\mathrm{Au})$, passes a large plateau in the energy range between 1.8 and $2.3 \mathrm{eV}$, covering the spectral position of 1,0 Mie plasmons in Au particles on $\mathrm{TiO}_{2}$. Because of the small dispersion of the dielectric function in this region, the resonance position of the plasmon mode is not fixed at a specific energy. This becomes intuitively clear by applying the plasmon resonance condition given in Eq. (1). Small modifications in the cluster environment, expressed by changes in its dielectric constant $\varepsilon_{m}$, shift the plasmon energy over the plateau region of the gold dielectric function. Such perturbations in the electromagnetic properties of the cluster surrounding are produced by interactions with the STM tip. ${ }^{7}$ Its polarizability increases the value of $\varepsilon_{m}$, thus shifting the resonance frequency of the collective mode from the Mie value of $2.3 \mathrm{eV}$ towards the opposite side of the plateau at $1.8 \mathrm{eV}$. For this reason, the actual position of the plasmon excitation crucially depends on the interaction strength between tip and cluster, which might cause the random distribution of observed emission lines from $\mathrm{Au} / \mathrm{TiO}_{2}$ in the plateau region.

In contrast to gold, the dielectric function of silver exhibits almost free electron-gas behavior in the energy range of Mie plasmons (Fig. 4). The strong dispersion of the polarizability stabilizes the collective mode in Ag particles around the Mie value, ${ }^{1,29}$ despite of an additional influence of the STM tip. The electromagnetic perturbations in the cluster surrounding lead to similar changes in the plasmon resonance condition, according to Eq. (1). However, because of the stronger frequency dependence of $\varepsilon_{1}(\mathrm{Ag})$, the energy 


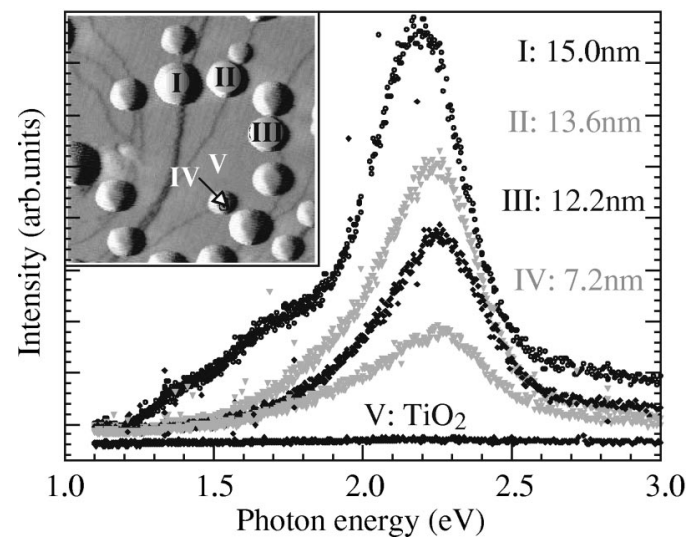

FIG. 5. Photon-emission spectra of differently sized Au particles on $\mathrm{TiO}_{2}\left(U_{\text {tip }}=-5 \mathrm{~V}, I=1.5 \mathrm{nA}\right)$. The emission is dominated by decays of Mie-like plasmons. The spectra can be assigned to clusters labeled in the STM image in the inset (size $130 \times 130 \mathrm{~nm})$.

position of the collective excitation is much less affected. Consequently, photon-emission measurements on Ag particles in a PSTM are less sensitive to modifications of the tip-sample coupling and give the intrinsic properties of $\mathrm{Ag}$ Mie plasmons.

\section{Cluster-size dependence of emission peaks}

As outlined above, the energy position of emission peaks from individual $\mathrm{Au}$ clusters on $\mathrm{TiO}_{2}$ is not directly correlated to corresponding cluster diameters, because of considerable tip influence on the system. This restriction could be weakened for series of photon-emission measurements, taken at one stable tip configuration. At these conditions, the relative dependence of the plasmon energy on cluster size becomes accessible to the experiment. However, the emission behavior must not be associated with the intrinsic optical properties of an $\mathrm{Au}$ cluster alone, because it is still altered by a constant interaction with the STM tip.

Figure 5 shows a series of photon-emission spectra for a number of differently sized $\mathrm{Au}$ particles on $\mathrm{TiO}_{2}$ (110). Each spectrum can be assigned to the cluster labeled in the STM topography in Fig. 5. The cluster-size dependence of peak positions, obtained from this experimental run, is displayed by black triangles in Fig. 6. Further data points from the same series have been added to the diagram. The peak energies around $2.3 \mathrm{eV}$ are characteristic for Mie plasmons in $\mathrm{Au}$ particles, where the collective mode is weakly modified by the presence of the STM tip (see discussion in Sec. III B). This interpretation is supported by the strong polarity dependence of measured peak heights, leading to intense emission peaks only for electron injection from the tip into the particle. In constrast, emission processes with considerable contribution of tip-induced plasmons are independent of the direction of tunneling electrons. ${ }^{7,15}$ With decreasing cluster sizes, emission peaks from $\mathrm{Au} / \mathrm{TiO}_{2}$ shift to higher photon energies. The size dependence $\hbar \omega(d)$ can be fitted with a functional relation proportional to the inverse cluster diameter $d^{-1}: \hbar \omega(d)=2.18 \mathrm{eV}+1.06 \mathrm{eV} \mathrm{nm} / d(\mathrm{~nm})$ (Fig. 6, upper solid line). Similar $d^{-1}$ correlations between plasmon

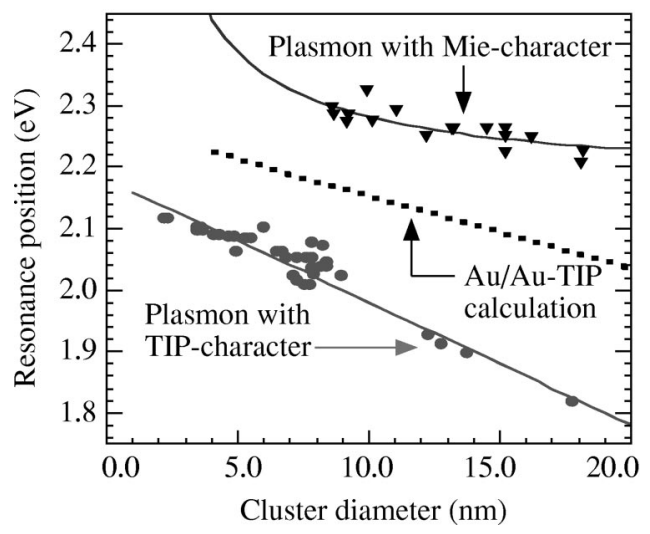

FIG. 6. Resonance positions vs cluster diameter for collective modes with strong TIP $(\mathbf{O})$ and $\mathrm{Mie}(\boldsymbol{\nabla})$ character in $\mathrm{Au} / \mathrm{TiO}_{2}$. Solid lines show fits of corresponding size dependences to experimental data. The broken line represents a calculation of TIP modes in an $\mathrm{Au} / \mathrm{Au}$ tunnel junction (for further details see text).

energy and cluster size have earlier been measured for Mie plasmons in ensembles of Ag particles [Ag/Ar (Ref. 32), $\mathrm{Ag} / \mathrm{Xe}$ (Ref. 33), Ag/vacuum ${ }^{34}$ ) and in individual Ag clusters on $\mathrm{Al}_{2} \mathrm{O}_{3} / \mathrm{NiAl}^{11}$ The effect was explained as a consequence of plasmon screening by localized $d$ electrons in the cluster, which leads to a reduction of the resonance energy. ${ }^{35}$ At the cluster surface, the depolarization influence of the $d$-electron cloud vanishes, because localized $d$ electrons cannot follow the spill-out tendency of delocalized $s, p$ electrons. With decreasing cluster size, the relative weight of unscreened surface-electrons increases, thus resulting in a higher plasmon frequency in smaller particles. The $d^{-1}$ behavior can, therefore, be understood as interplay between surface $\left(\propto d^{2}\right)$ and bulk effects $\left(\propto d^{3}\right)$ in small metal clusters. ${ }^{1}$ Corresponding blueshifts have also been found for Mie plasmons in Au cluster ensembles (e.g., Au particles in glass $\hbar \omega(d)=2.2 \mathrm{eV}+0.7 \mathrm{eVnm} / d(\mathrm{~nm})$ (Ref. 36) and in solution $\hbar \omega(d)=2.3 \mathrm{eV}+0.3 \mathrm{eVnm} / d(\mathrm{~nm})$ (Ref. 37). The size dependence of emission peaks around $2.3 \mathrm{eV}$, observed in the present STM experiment, reproduces the general trend in the plasmon energy shift as a function of cluster diameter. This agreement gives a further indication to assign the photon emission in this energy range to radiating decays of Mie-like plasmons in individual $\mathrm{Au}$ particles.

The size dependence of the photon-emission behavior changes completely, when the collective mode in an Au particle is strongly influenced by the presence of the STM tip. The tip polarizability shifts the plasmon resonance in the tunnel cavity to values around $1.8 \mathrm{eV}$. $^{38}$ The emitted intensity from $\mathrm{Au}$ particles becomes similar for positive and negative tunnel bias. ${ }^{15}$ Both observations indicate an increasing contribution of TIP modes to the emission process (see discussion in Sec. III B). For a representative series of measurements, energy positions of TIP-like plasmons are shown as a function of cluster diameter in Fig. 6 (filled circles). The spectra have been obtained after a transfer of gold particles from the surface to the tip apex at high electron currents. However, no changes in the tip configuration occurred during the course of measurements. For the given tip-sample coupling, the energy shift of the emission line can, therefore, be 
assigned to a relative cluster-size effect on the TIP. In contrast to Mie-like plasmons at $2.3 \mathrm{eV}$, plasmon peaks at lower photon energy and stronger TIP character do not follow a characteristic $d^{-1}$ size dependence. Peak energies decrease approximately proportional to cluster size and can be fitted by: $\hbar \omega(d)=2.3 \mathrm{eV}-0.02 \mathrm{eV} / \mathrm{nm} \times d(\mathrm{~nm})$. This modified size dependence demonstrates the physical nature of a collective electronic excitation, involving a considerable tip contribution in the STM cavity. At these conditions, the resonance frequency of the collective mode is no longer determined by intrinsic properties of the metal particle, but dominated by the size dependence of the tip-cluster interaction. For a qualitative description of the frequency shift as a function of cluster diameter, two effects have to be considered. (i) The dipole strength of the plasmon in the unperturbed cluster increases with diameter, thus leading to a larger image dipole in the STM tip and an enhanced dipole-dipole attraction. (ii) The tip-sample separation slightly reduces with decreasing cluster size, which additionally influences the dipole-dipole interaction according to Eq. (2).

To obtain an estimation of the size dependence of TIP modes in an $\mathrm{Au} / \mathrm{Au}$ tunnel junction, the Johansson model from Sec. III B was used..$^{30}$ The model allows the calculation of resonance frequencies for collective modes, formed between differently sized metal spheres and a planar electrode representing the STM tip. The estimation gives reasonable results, as long as the tip radius is much larger than the cluster diameter. For cluster radii below $10 \mathrm{~nm}$, calculated resonance positions of the TIP are shown as broken line in Fig. 6. The calculation reproduces the experimental trend of a TIP energy, linearly increasing with decreasing Au particle size. However, compared to measured energy positions of $\mathrm{Au}$ emission peaks, the simulation systematically overestimates the size-dependent TIP energies. This deviation can be attributed to the neglect of the $\mathrm{TiO}_{2}$ support in the theoretical model. In our experiment, the high polarizability of the oxide additionally shifts the plasmon peak to lower energies. A corresponding mechanism cannot be considered in the TIP model, leading to an artificial blueshift of collective modes in the calculation. On the other side, the reliability of the nonretarded model certainly breaks down in the limit of large metal spheres, equivalent to large Au particles. At these conditions, retardation effects of the electromagnetic potential become important, which in turn lead to a further redshift of the collective mode in the tip-sample system. ${ }^{39}$

\section{Line widths of emission peaks}

Experiments on photon emission from individual, $\mathrm{TiO}_{2}$ supported Au particles allow the determination of homogenous line widths without additional line broadening effects due to the cluster-size distribution on the surface. As discussed for the energy position of emission peaks, also line widths cannot be correlated to the cluster diameter alone, because of the variable-tip influence on electronic excitations in the particle. However, a strong and systematic correlation was observed between full-width at half-maximum (FWHM) and energy position of emission peaks, independently of the actual cluster size. Fig. 7 shows four emission spectra, mea-

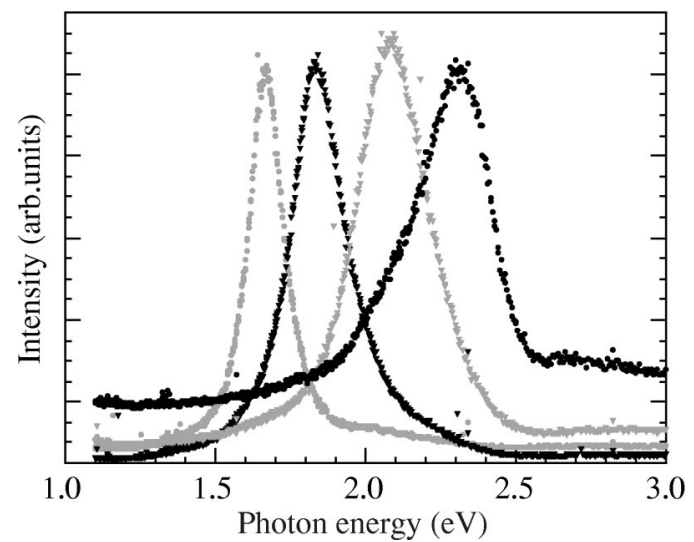

FIG. 7. Photon-emission spectra of $\mathrm{Au}$ particles on $\mathrm{TiO}_{2}\left(U_{\text {tip }}\right.$ $=-5 \mathrm{~V}, I=1.5 \mathrm{nA}$ ), measured with high spectral resolution of the spectrograph. Resonance positions result from an interplay between intrinsic cluster properties and interactions with the STM tip.

sured with high spectral resolution of the spectrograph. Peak positions result from an interplay of intrinsic cluster properties and tip-sample interactions and must not be assigned to cluster diameter alone. For decreasing peak energies, a considerable narrowing of the line widths is observed. Whereas photon-emission peaks with strong Mie character around 2.3 $\mathrm{eV}$ display an FWHM of approximately $300 \mathrm{meV}$, the homogeneous line width is reduced to $125 \mathrm{meV}$ for TIP-like modes at $1.8 \mathrm{eV}$. Based on the uncertainty relation, the narrowing of the emission lines can be interpreted as a lifetime prolongation of the collective excitations from 4.2 to 10.2 fs. ${ }^{40}$ The determination of line widths as a function of resonance frequency is reproducible and almost independent of the actual tip configuration. Figure 8 shows the increase of FWHM with energy of the emission peaks, derived from numerous measurements. Plasmon line widths for gold particles, embedded in a $\mathrm{TiO}_{2}$ matrix ${ }^{41}$ and in solution, ${ }^{37}$ have been added for comparison. The data point obtained for $\mathrm{Au}$

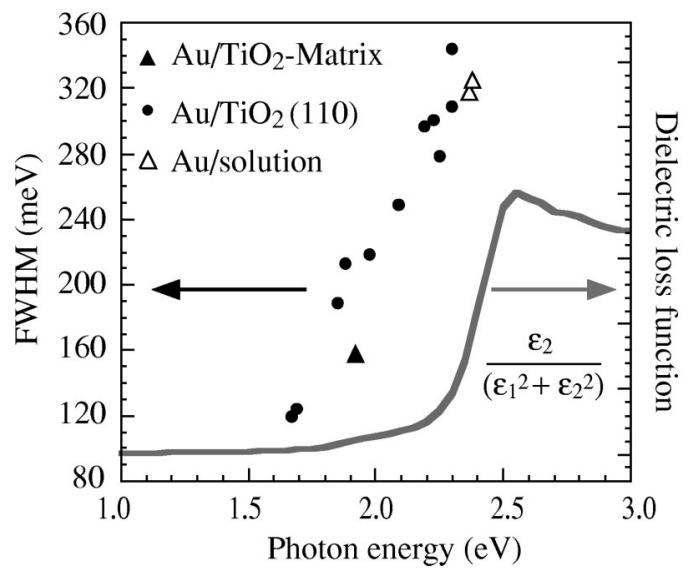

FIG. 8. FWHM vs resonance position for emission peaks from $\mathrm{Au} / \mathrm{TiO}_{2}$. Data points have been added for Au particles in a $\mathrm{TiO}_{2}$ matrix (Ref. 41) and in solution (Ref. 37). The solid line represents the bulk dielectric loss function for gold, calculated from data in Ref. 26. 
in a $\mathrm{TiO}_{2}$ matrix was derived from a scanning nearfield optical microscope experiment; clusters in solution were investigated by extinction cross-section spectroscopy.

Since the Au particle size neither exclusively determines position nor width of the corresponding emission line, cluster-size effects cannot be considered to interpret the observed linewidths, although they certainly have a contribution. Therefore, our explanation concentrates on bulk damping processes, responsible for the decrease of plasmon lifetime and the line broadening. A variety of decay mechanisms has to be considered for collective modes in the STM cavity. ${ }^{1,7,29}$ Inside an $\mathrm{Au}$ particle, dielectric losses or electron-phonon scattering cause a dephasing and decay of the plasmon mode. Because of the large time constants for electron-phonon coupling in the order of $\tau_{\text {Phonon }}>100 \mathrm{fs}$, this contribution can be neglected for the total lifetime. ${ }^{42}$ The emission of photons from an oscillating dipole leads to a weakening of the dipole strength and gives rise to the radiation damping. The corresponding decay time is dominated by resonance frequency $\left(\omega_{\mathrm{Pl}}\right)$ and mean dipole length $\left(d_{\mathrm{Au}}\right)$ according to ${ }^{40}$

$$
\tau_{\mathrm{Rad}}=\frac{3}{2} * \frac{c^{3}}{d_{\mathrm{Au}}^{3} \omega_{\mathrm{PL}}^{4}} .
$$

For an Au cluster of $10 \mathrm{~nm}$ diameter and a plasmon energy of $2.3 \mathrm{eV}$, the calculated lifetime contribution of $\tau_{\mathrm{Rad}}>270 \mathrm{fs}$ is also far too long to explain the broad homogenous line widths observed for Au particles on $\mathrm{TiO}_{2}$. Additional decay channels may be opened by plasmon interactions with the $\mathrm{TiO}_{2}$ substrate and the $\mathrm{STM}$ tip. $\mathrm{TiO}_{2}$-mediated damping can essentially be ruled out, because the maximum plasmon energy of $2.3 \mathrm{eV}$ is not sufficient to excite electron-hole pairs in the $3 \mathrm{eV}$ band gap of $\mathrm{TiO}_{2}$. The importance of tip-sample interactions on the lifetime of collective modes cannot be estimated in an easy manner. However, with increasing tip coupling, responsible for the low-energy TIP modes, an increase of the tip-induced damping efficiency is expected, which is in contrast to experimental observations.

These arguments suggest a concentration on electronelectron scattering processes inside an Au particle to explain the short plasmon lifetimes. ${ }^{43}$ Bulk dielectric losses are described by the imaginary part of the gold dielectric function $\left(\varepsilon_{2}\right)$, which includes absorption in the free electron gas and energy dissipation due to interband transitions. ${ }^{26}$ Surface scattering processes as a function of cluster size are not considered. ${ }^{29}$ In a rough estimation, the damping efficiency due to bulk dielectric losses becomes proportional to $\varepsilon_{2}(\omega) /\left(\varepsilon_{1}{ }^{2}+\varepsilon_{2}{ }^{2}\right) .{ }^{40}$ The corresponding functional relation has been added to Fig. 8. The strong increase in the electronelectron-damping efficiency at energies above $2.0 \mathrm{eV}$ reproduces the trend in the measured FWHM for $\mathrm{Au} / \mathrm{TiO}_{2}$. The qualitative agreement gives a strong indication of the dominant plasmon decay channel, determined by dielectric losses in the Au particle.

Also from a microscopic point a view, the enhancement of plasmon damping with increasing resonance frequency can be rationalized. The electronic structure of bulk gold is characterized by the onset of the gold $d$ bands with a high density of states at $2.3 \mathrm{eV}$ below the Fermi level. ${ }^{44}$ In this energy region, strong absorption occurs due to interband transitions from the $d$ bands to unoccupied states above the Fermi level. The necessary energy quantum can be provided by the plasmon oscillation, resulting in enhanced damping and broad emission lines of the collective mode. The probability of a plasmon decay increases as its energy approaches the interband transitions at $2.3 \mathrm{eV}$. The model is compatible with the short lifetime of collective modes with strong Mie character at around $2.3 \mathrm{eV}$ compared to TIP-like modes at $1.8 \mathrm{eV}$. However, a determination of absolute time constants for the different plasmon decay channels demands for more sophisticated theoretical models and is beyond the scope of this paper.

\section{CONCLUSIONS}

The present experiments demonstrate the importance of tip-sample interactions for collective electronic modes in small Au particles, excited with the local electron beam from an STM tip. Whereas electronic properties of idealized cluster-substrate systems are dominated by Mie-plasmon excitations, the presence of a polarizable metal tip in proximity to the cluster considerably redshifts the resonance frequency of the collective mode. For single $\mathrm{TiO}_{2}$ supported Au particles, a transition from Mie-like electronic excitations centered at around $2.3 \mathrm{eV}$ to TIP-like plasmon modes at $1.8 \mathrm{eV}$ has been observed as a function of tip-sample interaction and microscopic tip configuration. This variability in the emission behavior of Au particles is explained by a peculiarity of the gold dielectric function, which passes an extended plateau in the energy range between 1.8 and $2.3 \mathrm{eV}$. The negligible dispersion makes the plasmon resonance in Au particles sensitive to external electromagnetic perturbations, as induced by the presence of the STM tip. Conclusively, STMmediated photon-emission spectroscopy cannot directly be employed to investigate optical properties of individual $\mathrm{Au}$ particles on $\mathrm{TiO}_{2}$. In this case, the measured resonance frequencies of collective excitations results from an interplay between intrinsic cluster properties and interactions with the tip in the STM cavity. For materials characterized by a strong dispersion in their dielectric function, such as silver, the resonance frequency of collective modes is stabilized close to the Mie value and less sensitive to tip-sample interactions. For these systems, photon emission peaks measured in a PSTM can indeed be correlated with intrinsic optical properties of the metal particles.

\section{ACKNOWLEDGMENTS}

For financial support we are grateful to the Deutsche Forschungsgemeinschaft, the Fond der Chemischen Industrie and the NEDO Research Grant on Photon and Electron Controlled Surface Processes. 
*Corresponding author. Present address: University of California, Irvine, 4129 Frederick Reines Hall, Irvine, CA 92697. FAX: (949)-824-2174. Email address: nilius@fhi-berlin.mpg.de

${ }^{1}$ U. Kreibig and W. Vollmer, Optical Properties of Metal Clusters, Springer Series Materials Science Vol. 25, (Springer, Berlin, 1995).

${ }^{2}$ F. Stietz and F. Träger, Philos. Mag. B 79, 1281 (1999).

${ }^{3}$ M. Kaempfe, H. Hofmeister, S. Hopfe, G. Seifert, and H. Graener, J. Phys. Chem. B 104, 11847 (2000).

${ }^{4}$ G. I. Stegeman and E. M. Wrigth, Opt. Quantum Electron. 22, 95 (1990)

${ }^{5}$ J. Kunckel, Ars Vitrria Experimentalis oder die vollkommene Glasmacher-Kunst (Frankfurt, 1689).

${ }^{6}$ J. H. Coombs, J. K. Gimzewski, B. Reihl, and J. K. Sass, J. Microsc. 152, 325 (1988).

${ }^{7}$ R. Berndt, in Scanning Probe Microscopy, edited by R. Wiesendanger, Springer Series Nanoscience and Technology (Springer, Berlin, 1998) 97.

${ }^{8}$ P. Dumas, C. Syrykh, I. Makarenko, and F. Salvan, Europhys. Lett. 40, 447 (1997).

${ }^{9}$ A. Downes and M. E. Welland, Appl. Phys. Lett. 72, 2671 (1998).

${ }^{10}$ N. Nilius, N. Ernst, and H.-J. Freund, Chem. Phys. Lett. 349, 351 (2001)

${ }^{11}$ N. Nilius, N. Ernst, and H.-J. Freund, Phys. Rev. Lett. 84, 3994 (2000).

${ }^{12}$ A. Olkhovets, S. Evoy, and H. G. Craighead, Surf. Sci. Lett. 453, L299 (2000).

${ }^{13}$ N. Nilius, N. Ernst, and H.-J. Freund, Surf. Sci. Lett. 478, L327 (2001).

${ }^{14}$ A. Downes, M. E. Taylor, and M. E. Welland, Phys. Rev. B 57, 6706 (1998).

${ }^{15}$ R. Berndt, J. K. Gimzewski, and P. Johansson, Phys. Rev. Lett. 67, 3796 (1991).

${ }^{16}$ P. Johansson, R. Monreal, and P. Apell, Phys. Rev. B 42, 9210 (1990).

${ }^{17}$ M. Ando, T. Kobayashi, and M. Haruta, Catal. Today 36, 153 (1993).

${ }^{18}$ G. R. Bamwenda, S. Tsubota, T. Nakamura, and M. Haruta, Catal. Lett. 44, 83 (1997).

${ }^{19}$ M. Valden, X. Lai, and D. W. Goodman, Science 281, 1647 (1998)

${ }^{20}$ M. Mavrikakis, P. Stoltze, and J. K. Norskov, Catal. Lett. 64, 101 (2000).

${ }^{21}$ N. Nilius, A. Cörper, G. Bozdech, N. Ernst, and H.-J. Freund, Prog. Surf. Sci. 67, 99 (2001).
${ }^{22}$ R. Hasiguti, Adv. Mater. Sci. 2, 69 (1972).

${ }^{23}$ H. Onishi and Y. Iwasawa, Phys. Rev. Lett. 76, 791 (1996).

${ }^{24}$ R. E. Tanner, M. R. Castell, and G. A. D. Briggs, Surf. Sci. 412, 672 (1998).

${ }^{25}$ J. Pascual, J. Camassel, and H. Mathieu, Phys. Rev. B 18, 5606 (1978).

${ }^{26}$ Handbook of Optical Constants of Solids, edited by E. D. Palik (Academic, Orlando, 1985).

${ }^{27}$ G. Mie, Ann. Phys. (Leipzig) 25, 377 (1908); R. Gans, ibid. 37, 881 (1912).

${ }^{28}$ J. B. Goodenough and M. Hamnett, in Physics of Binary Compounds, edited by O. Madelung, M. Schulz, and H. Weiss, Landolt Börnstein, New Series, Group III, Vol. 17, Pt. g (Springer, Berlin, 1984), p. 142.

${ }^{29}$ H. Hövel, S. Fritz, A. Hilger, U. Kreibig, and M. Vollmer, Phys. Rev. B 48, 18178 (1993).

${ }^{30}$ P. Johansson and R. Monreal, Z. Phys. B: Condens. Matter 84, 269 (1991)

${ }^{31}$ E. Anisimovas and P. Johansson, Phys. Rev. B 59, 5126 (1999).

${ }^{32}$ W. Ekardt, D. Tran Thoai, F. Frank, and W. Schulze, Solid State Commun. 46, 571 (1983).

${ }^{33}$ K.-P. Charlé, L. König, S. Nepijko, I. Rabin, and W. Schulze, Cryst. Res. Technol. 33, 1085 (1998).

${ }^{34}$ J. Tiggesbäumker, L. Köller, K. H. Meiwes-Broer, and A. Liebsch, Phys. Rev. A 48, R1749 (1993).

${ }^{35}$ A. Liebsch, Phys. Rev. B 48, 11317 (1993).

${ }^{36}$ U. Kreibig, J. Phys. 38, 2 (1977).

${ }^{37}$ J. Hodak, A. Henglein, and G. Hartland, J. Chem. Phys. 112, 5942 (2000).

${ }^{38}$ K. Ito, S. Ohyama, Y. Uehara, and S. Ushioda, Surf. Sci. 324, 282 (1995).

${ }^{39}$ P. Johansson, Phys. Rev. B 58, 10823 (1998).

${ }^{40}$ E. J. Heilweil and R. M. Hochstrasser, J. Chem. Phys. 82, 4762 (1985).

${ }^{41}$ T. Klar, M. Perner, S. Grosse, G. v. Plessen, W. Spirkl, and J. Feldmann, Phys. Rev. Lett. 80, 4249 (1998).

${ }^{42}$ J. Y. Bigot, J.-C. Merle, O. Cregut, and A. Daunois, Phys. Rev. Lett. 75, 4702 (1995).

${ }^{43}$ M. Perner, P. Bost, U. Lemmer, G. v. Plessen, J. Feldmann, U. Becker, M. Mennig, M. Schmitt, and H. Schmidt, Phys. Rev. Lett. 78, 2192 (1997).

${ }^{44}$ N. E. Christensen and B. O. Seraphin, Phys. Rev. B 4, 3321 (1971). 